\title{
The European Association for the Study of Obesity (EASO) Endorses the Milan Charter on Urban Obesity
}

\author{
Michele O. Carruba ${ }^{a}$ Luca Busetto ${ }^{b, c}$ Sheree Bryant ${ }^{d}$ Antonio Caretto $^{e}$ \\ Nathalie J. Farpour-Lambert ${ }^{f}$ Giuseppe Fatati ${ }^{\mathrm{g}}$ Diego Foschi ${ }^{\text {h }}$ \\ Francesco Giorgino $^{i}$ Jason C.G. Halford ${ }^{j}$ Andrea Lenzi ${ }^{k}$ Giuseppe Malfil \\ Grace O'Malley $^{\mathrm{m}} \mathrm{n}$ - David Napier $^{\mathrm{O}}$ Ferruccio Santini $^{\mathrm{p}}$ Paolo Sbraccia ${ }^{\mathrm{q}}$ \\ Chiara Spinato $^{r}$ Euan Woodward ${ }^{n}$ Enzo Nisoli ${ }^{a}$
}

\begin{abstract}
${ }^{a}$ Center for Study and Research on Obesity, Department of Biomedical Technology and Translational Medicine, University of Milan, Milan, Italy; ${ }^{\mathrm{b}}$ Department of Medicine, University of Padova, Padova, Italy; ${ }^{\mathrm{C}}$ Center for the Study and the Integrated Management of Obesity, Padova University Hospital, Padova, Italy; ${ }^{\mathrm{d}}$ The European

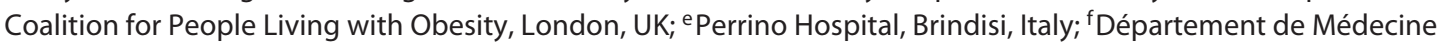
Communautaire, de Premier Recours et des Urgencies, Hôpital de Beau-Séjour, Hôpitaux Universitaires de Genève, Geneve, Switzerland; 9 Unit of Diabetology, Dietology and Clinical Nutrition, S. Maria Hospital, Terni, Italy; ${ }^{\mathrm{h}}$ Department of Biomedical and Clinical Sciences, University of Milan, Milan, Italy; ${ }^{\mathrm{i}}$ Department of Emergency and Organ Transplantation, University of Bari Aldo Moro, Bari, Italy; ${ }^{j}$ School of Psychology, University of Leeds, Leeds, UK; ' Department of Experimental Medicine, Sapienza University of Rome, Rome, Italy; 'Department of Dietetics and Clinical Nutrition, San Giovanni Battista Hospital, Turin, Italy; ${ }^{m}$ Division of Population Health, RCSI University of Medicine and Health Sciences, Dublin, Ireland; ${ }^{n}$ European Association for the Study of Obesity,

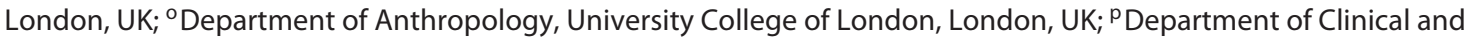
Experimental Medicine, University Hospital of Pisa, Pisa, Italy; ${ }^{9}$ Department of Systems Medicine, University of Rome

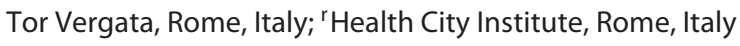

\section{Keywords}

Non-communicable diseases · Nutrition · Obesity ·

Prevention - Urban health

\section{Abstract \\ The Milan Charter on Urban Obesity highlights the challeng- es of urban environments as a battleground for human health, as cities are often organized to subvert public health goals, and promote rather than prevent the development of obesity and consequent non-communicable diseases. The}

Charter articulates ten principles which detail actions and strategies through which general practitioners, diverse medical specialists, related healthcare professionals, administrators and healthcare practice managers, policy actors within health systems and at a national level - along with experts across disciplines, and citizens, can work in cooperation to meet this challenge and improve public health. The Charter urges the adoption of decisions that deliver the following: (i) policies which enable our cities to become healthier and less obesogenic, more supportive of well-being and less health-disruptive in general, and (ii) policies that fully karger@karger.com www.karger.com/ofa

Karger $\stackrel{\text { ' }}{5}$

GOPEN ACCESS
(C) 2021 The Author(s)

Published by S. Karger AG, Basel

This article is licensed under the Creative Commons AttributionNonCommercial-NoDerivatives 4.0 International License (CC BY NC-ND) (http://www.karger.com/Services/OpenAccessLicense). Usage and distribution for commercial purposes as well as any distribution of modified material requires written permission.
Michele O. Carruba

Department of Biomedical Technology and Translational Medicine University of Milan, via Vanvitelli 32

IT-20129 Milan (Italy)

michele.carruba@unimi.it 
support primary prevention strategies, that address social stigma, and that ensure fair access to treatment for people living with obesity. The Milan Charter on Urban Obesity aims to raise awareness of our shared responsibility for the health of all citizens, and focuses on addressing the health of people living with obesity - not only as a challenge in its own right, but a gateway to other major non-communicable diseases, including cardiovascular diseases, type 2 diabetes, and some cancers.

(c) 2021 The Author(s)

Published by S. Karger AG, Basel

\section{Introduction}

In 1999, on the occasion of the 9th annual European Congress on Obesity organized by the Center for the Study and Research on Obesity (CSRO) at the University of Milan in collaboration with the European Association for Study of Obesity (EASO), the "Milan Declaration" was presented. The declaration was reaffirmed at the World Exposition Milano 2015 (EXPO 2015), which was attended by 140 countries [1]. This represented the first Call to Action issued by European experts for governments to recognize obesity as a chronic disease as a matter of urgency, highlighting the importance of treating obesity with appropriate evidence-based therapies. The Call to Action established that by reducing the incidence and prevalence of this disease by one percentage point, we could potentially avoid 1-3 million cases of type 2 diabetes, cardiovascular diseases including high blood pressure, and some cancers among European citizens - an estimate that could rise to $2-9$ million cases with a $5 \%$ reduction in prevalence [2].

Urban environments, including natural resources found within city boundaries like clean air and water, along with land use policies, infrastructure development, and uses of the built environment, have a monumental impact on individual and community well-being [3]. Acknowledgement of these interdependencies should be central features orienting the development and implementation of urban public policies [4]. The relationships between health, quality of life, and the environment are crucial in empowering citizens to participate fully in society [5]. Similarly, acknowledging and understanding these linkages are important for those working in social and medical sciences, who endeavor to improve public health and cultivate human potential [6].

Moving beyond purely classifying disease and disorders, the WHO International Classification of Functioning, Disability and Health (ICF; https://www.who.int/ classifications/icf/en/) addresses these relationships effectively using a universal lexicon. The global increase of non-communicable diseases, including obesity and type 2 diabetes, is influenced by higher urbanization levels, along with population ageing, disordered sleep patterns, sedentary lifestyles, social inequalities, and the promotion and consumption of unhealthy food. These aspects of the urban context have been described across official national and international documents (European Commission, https://ec.europa.eu/health/non_communicable_diseases/overview_en; WHO, https://www.who.int/ global-coordination-mechanism/ncd-themes/povertydevelopment/en/) [7, 8].

The "Carta di Milano," signed during the 2015 Milan EXPO, sets forth key principles and objectives focused on improving healthy diets, sustainable development, and environmental sustainability while upholding human rights. The Charter was translated into 19 languages and was presented to the United Nations Secretary General Ban Ki-moon on October 16, 2015. The "Carta di Milano" asked signatories to undertake explicit commitments regarding the right to food - one of the fundamental human rights enshrined in Article 25 of the Universal Declaration of Human Rights.

The manifesto "Health in the City: The Common Good," promoted by The Health City Institute, was adopted in 2016. This document defines the main drivers for cities to review and implement to improve the determinants of health in urban environments and sets forth strategies to improve citizens' lifestyles and overall health [4]. In 2017, during the G7 summit, the Italian Ministry of Health and ANCI (the National Association of Italian Municipalities) signed the "Roma Urban Health Declaration," a document which defined strategic actions to improve health in cities using a holistic approach viewed through the lens of both the individual and relevant institutions, taking a multisectoral approach to the promotion of health policies in cities [9]. The "Manifesto on Obesity," promoted by the Italian Obesity Network (IO-NET) and signed in 2018, describes a roadmap to improving quality of life for people living with obesity [10]. The "Charter of Rights and Duties to People with Obesity" was also promoted by IO-NET and was signed in 2019; the Charter reiterates that the rights of people living with obesity are identical to the human and social rights of all citizens. These rights include equal access to information, therapeutic education, diagnosis and treatment of obesity, and control of complications arising from obesity. In 2019, this series of measures culminated with the Italian Chamber of Deputies unanimously adopting a "Motion 
on Obesity" to empower government with the tools necessary for the prevention and effective treatment of obesity. Developing a national plan is amongst these commitments, which aim to standardize activities addressing: the prevention and management of obesity; access to diagnostic procedures and management of complications; access to evidence-based nutritional interventions, and, where appropriate, full access to second-level specialist centers to access multidisciplinary psychological, pharmacological, and surgical approaches to care [11].

The sum of these documents has inspired the creation of a Sustainability Pact for our cities. This will help address challenges linked to obesity and the multisectoral management of this complex chronic disease. This pact, which originated in the city of Milan, is thus named the Milan Charter on Urban Obesity.

\section{Statement and Action}

The Milan Charter on Urban Obesity is based on the development of ten key principles that aim to qualify the action and the strategies as a pact between health professionals, scientists, health managers, policy makers, administrators, and citizens. The Charter urges political decisions that guarantee achievement of the fundamental objectives of fair access to treatment, of primary prevention interventions to address social stigma and discrimination, and of making our cities healthier and less health disrupting environments, in order to create a fair and sustainable future for people with obesity.

The text of the Milan Charter on Urban Obesity is the following:

We, women and men, administrators, physicians, health professionals, scientists, coalitions for people living with obesity, and citizens endorse this document known as the Milan Charter on Urban Obesity, to commit to specific actions, to address obesity as a disease, in full synergy, in order to ensure a better quality of life for people with obesity living in large urban areas.

We contend that multilateral joint efforts are required to make the urban context and the environment less health disrupting and more focused on the quality of life of individuals living with obesity, and this will be achieved by removing social, architectural, structural, and cultural barriers that prevent people living with obesity from leading a healthy life.

We believe that the synergistic work accomplished by multiple stakeholders will lead to truly innovative and creative efforts enabling strategies, processes and resulting actions to overcome the major challenges linked to obesity, such as access to treatments, implementation of effective prevention interventions, and to address social stigma and discrimination, in large urban and metropolitan areas.

By signing the Milan Charter on Urban Obesity:

We AFFIRM the assumption of responsibility on our part to implement daily actions and choices that protect the right to health of people with obesity;

We PLEDGE to solicit political decisions at all levels that guarantee the achievement of the fundamental objective of fair access to treatment, to start primary prevention interventions to address social stigma and to make our cities healthier and less health disrupting.

We believe it is necessary:

TO GUARANTEE full equitable access to medical and scientific information, age-appropriate care, and innovative treatments to individuals living with obesity in the entire urban territory;

TO SUPPORT people with obesity and their family members in overcoming obstacles, prejudice, mistrust, and discrimination;

TO ENCOURAGE institutions to urgently consider obesity as a disease that can cause significant disability and that requires specific legislative and regulatory instruments particularly for the protection of those most vulnerable;

TO REMOVE architectural barriers in cities, medical centers, transportation and mobility infrastructures, workplaces, education and meeting places, preventing people with obesity from participating fully in life;

TO ADOPT policies aimed at reducing the supply and marketing of ultra-processed food and beverages contributing to obesity and improving accessibility to healthy food;

TO PROMOTE nutritional education and practical skills training, in schools and through press and communication messaging;

TO DISSEMINATE accurate, well-balanced and nonpartisan information, scientifically evidence-based, with proper advertising and public service announcements involving each ministry;

TO INTRODUCE urban planning policies and protocols aimed at improving healthy urban environments providing more access to safe green spaces and outdoor equipment/games for exercising as an integral part of daily life, thus ensuring their feasibility; 
TO SUPPORT access for all citizens to clean water, air and soil in terms of pollution and aligning to environmental sustainability and the aims of the United Nations Environment Programme and the Global Pact for the Environment;

TO SUPPORT full access to multidisciplinary care to improve the quality of life of individuals with obesity, and reduce the risk of disabling and/or fatal complications;

TO CONSIDER prevention and reduction of obesity as an urban policy objective ensuring that actions are taken in all sectors: health, education, transport, economic, media and culture, sustainable development and social services;

TO INCREASE the involvement of institutions, general practitioners and specialist physicians, health professionals, citizens and media, urging them to recognize the severity of obesity as a disease;

TO ADOPT policies to protect people, employees, and students respecting the individual regardless of his/her shape, size or weight, avoiding discrimination in society, schools, and universities, in job interviews, and countering all types of bullying and social disparity;

TO CONSIDER the individual with obesity as a person and not only as a patient, by using the term "people living with obesity" and not "obese people," making sure that media do not use false and inaccurate stereotypes of obesity or images that undermine, devalue or dehumanize.

We believe it is unacceptable that:

The person living with obesity is often a victim of prejudice and is subject to discrimination;

The person living with obesity is a victim of cultural stereotypes, fueled by different social contexts; dehumanizing images which often accompany news stories about obesity, both in social media and in advertising campaigns using posters in cities, that portray the person living with obesity in a negative way; in many urban contexts the person living with obesity does not get adequate attention regarding his/her health.

At the same time, we are willing to contribute to leaving a healthier and more equitable and sustainable world to future generations, and as citizens, we are committed to:

ADOPTING healthier lifestyles;

AVOIDING waste of food and water in all our daily activities, both at home and in external contexts;

ADOPTING responsible behavior and practices in order to protect the environment;
PROMOTING nutritional and environmental education in the family setting for a more conscious growth of new generations;

CHOOSING food supplies consciously, considering the impact of their production chain on environmental sustainability;

BEING an active partner in building more sustainable cities through innovative solutions stemming from our professional competencies, creativity, and talent.

As members of the civil society and part of the community, we are committed to:

MAKING OUR VOICE HEARD at all decision-making levels, for more livable and equitable cities;

REPRESENTING the demands of the civil society in debates and public policy processes to address obesity as a health and societal priority;

REPORTING all forms of stigma and discrimination towards people living with obesity;

WORKING with city and health institutions to remove architectural barriers from public buildings, public transport systems, sports facilities, schools, workplaces, and medical centers;

FOSTERING nutritional and environmental education to reach collective awareness on their importance for the future of humanity.

By underwriting the Milan Charter on Urban Obesity, we, health professionals, administrators, physicians, experts, coalitions for people living with obesity, and citizens, call on governments and mayors to:

ADOPT regulations and laws to ensure the right to evidence-based clinical treatment for all persons living with obesity;

STRENGTHEN the laws caring for people living with obesity and their family members;

PROMOTE the debate on obesity and bio-psychosocial drivers of health and healthy lifestyles in all cultural settings;

SUPPORT and spread the culture of healthy sustainable food and beverages as a tool for global public health;

PROMOTE a pact on nutritional strategies, both in urban and rural contexts, concerning sustainable and equitable access to healthy food;

INCREASE resources allocated to research on metabolic disorders (including obesity and diabetes) and how their effects and treatments can be better understood using the WHO ICF framework, and transfer and disseminate results in the education and communication fields; 
INTRODUCE or strengthen programs of nutritional, physical and environmental education in schools and school canteens, to be used as health and prevention instruments, by enhancing particularly the knowledge and exchange of food cultures, starting from typical, organic and local "slow food";

DEVELOP measures and policies for national and regional public health systems, promoting healthy, equitable and sustainable nutrition and reducing nutritional deficiencies;

PROMOTE information campaigns aimed at citizens' increase of awareness about the relevance and the risks linked to overweight and obesity and the solutions that are available for obesity management;

INVEST in building an environment and an urban social context which is less health disrupting, by developing policies and infrastructures devoted to monitoring interventions addressing the aim.

Since we believe that effective efforts to address obesity and its spread has to start from cities, we are committed to adopting the principles and practices enshrined in this Milan Charter on Urban Obesity, turning them into an active part of our pledge for the future of humanity and our planet.

A fair and sustainable future for people with obesity lies in our responsibility, too.

\section{Conclusion}

The impact of this document will depend on its adoption and on the uptake of these recommended policies, as well as on the comprehensive adherence to the actions within. We encourage the widest possible dissemination in order to reach: (i) policy makers who care about the public good, (ii) health authorities attentive to the needs of various territories, (iii) urban planners open to innovative sustainable development strategies, and (iv) most im- portantly citizens, many of whom will be people living with obesity. This ambitious plan will engage all actors in the urban ecosystem in developing a collaborative framework to secure the long-term health for all of our citizens. Initiating a resolution process applying a public health lens to urban policies will set this in motion.

\section{Acknowledgements}

The pact described in this paper is called the Milan Charter on Urban Obesity and has been promoted by the Center for the Study and Research on Obesity (CSRO) of the University of Milan, in cooperation with the EASO (European Association for the Study of Obesity), the Municipality of Milan, the Region of Lombardy, ANCI (National Association of Italian Municipalities), the Parliamentary Intergroup on Obesity and Diabetes, HCI (Health City Institute), CCD (Cities Changing Diabetes) network, IO-NET (Italian Obesity Network), OPEN (Obesity Policy Engagement Network), SIO (Italian Obesity Society), SIP (Italian Society of Paediatrics), SIEDP (Italian Society of Paediatric Endocrinology and Diabetology), ADI (Italian Association of Dietetics and Clinical Nutrition), IBDO Foundation (Italian Barometer Diabetes Observatory), SIMG (Italian Society of General Medicine and Primary Care), Amici Obesi onlus (Friends of $\mathrm{PwO}$ association), and CittadinanzAttiva (Active Citizenship Association).

\section{Conflict of Interest Statement}

The authors have no conflicts of interest to declare.

\section{Funding Sources}

The authors declare no funding relevant in the preparation of this manuscript.

\section{Author Contributions}

M.O.C. and E.N. conceived the study. All authors discussed the study. M.O.C. and E.N. drafted the manuscript. L.B., S.B., J.C.G.H., G.O., D.N., and E.W. revised the manuscript. All authors approved the final version of the manuscript.

\section{References}

1 Frühbeck G, Sbraccia P, Nisoli E, Woodward E, Yumuk V, Farpour-Lambert NJ, et al. 2015 Milan declaration: a call to action on obesity - an EASO position statement on the occasion of the 2015 EXPO. Obes Facts. 2016;9(4)2968.

2 Swinburn BA, Kraak VI, Allender S, Atkins VJ, Baker PI, Bogard JR, et al. The global syndemic of obesity, undernutrition, and climate change: The Lancet Commission report. Lancet. 2019:393:791-846.

3 Kiplinger [Internet]. 10 best cities for the next decade [cited 2020 Nov 28]. Available from: https://www.kiplinger.com/article/business/ t006-c000-s002-10-best-cities-for-the-nextdecade.html.

4 Lenzi A, Capolongo S, Ricciardi G, Signorelli C, Napier D, Rebecchi A, et al. New compe- tences to manage urban health: health City Manager core curriculum. Acta Biomed. 2020 Apr;91 3-S:21-8. Available from: https:// www.mattioli1885journals.com/index.php/ actabiomedica/article/view/9430.

5 Castán Broto V, Bulkeley H. A survey of urban climate change experiments in 100 cities. Glob Environ Change. 2013 Feb;23(1):92102 . 
6 Bettencourt L, West G. A unified theory of urban living. Nature. 2010 Oct;467(7318):9123.

7 Wyse CA, Celis Morales CA, Graham N, Fan $\mathrm{Y}$, Ward J, Curtis AM, et al. Adverse metabolic and mental health outcomes associated with shiftwork in a population-based study of 277,168 workers in UK biobank $[\{\mathrm{LT}\}]$ sup/ [\{GT\}]. Ann Med. 2017 Aug;49(5):411-20. Available from: https://pubmed.ncbi.nlm. nih.gov/28166415/.
8 OHCHR [Internet]. Special rapporteur on human rights and the environment. [cited 2020 Nov 28]. Available from: https://www. ohchr.org/en/Issues/environment/SRenvironment/Pages/SRenvironmentIndex.aspx.

9 Ministero della Salute [Internet]. Urban Health Rome Declaration [cited 2020 Nov 28]. Available from: http://www.salute.gov.it/ imgs/C_17_EventiStampa_501_intervisteRelatori_itemInterviste_0_fileAllegatoIntervista.pdf.
10 Italian Obesity Network [Internet]. Manifesto Dell'Italian Obesity Network Per Un Futuro Sostenibile [cited 2020 Nov 28]. Available from: https://www.obesityday.org/usr_files/ home/manifesto-firmato.pdf.

11 Brunani A, Raggi A, Sirtori A, Berselli ME, Villa V, Ceriani F, et al.; ICF-OBESITY Group. An ICF-based model for implementing and standardizing multidisciplinary obesity rehabilitation programs within the healthcare system. Int J Environ Res Public Health. 2015 May;12(6):6084-91. 